\title{
Transcranial magnetic stimulation studies in the Miller Fisher syndrome: evidence of corticospinal tract abnormality
}

\author{
Y L Lo, P Ratnagopal
}

\begin{abstract}
Objectives-To evaluate serial central motor conduction time in the Miller Fisher syndrome.

Method-Three patients with classic Miller Fisher syndrome were evaluated clinically. They had serial central motor conduction times measured with transcranial magnetic stimulation and nerve conduction studies. Motor evoked potentials were recorded from the first dorsal interossei and abductor hallucis muscles. Results-All three patients showed reduction in central motor conduction times in tandem with gradual clinical improvement at each review.

Conclusions-There is electrophysiological evidence of a central reversible corticospinal tract conduction abnormality in the Miller Fisher syndrome.

(F Neurol Neurosurg Psychiatry 2001;71:210-214)
\end{abstract}

Keywords: Miller Fisher syndrome; central motor conduction time; corticospinal tract abnormality

The Miller Fisher syndrome, clinically defined by the triad of ataxia, areflexia, and ophthalmoplegia, is an uncommon form of acquired inflammatory demyelinating polyneuropathy. ${ }^{1}$ An immunological process ${ }^{2-4}$ is likely to be involved in its pathogenesis akin to the more common forms of axonal or demyelinating Guillain-Barré syndrome, but its exact site of abnormality remains poorly defined in terms of clinical, radiological, or neurophysiological evidence. In particular, there is minimal evidence to suggest upper motor corticobulbar or corticospinal tract involvement. The disease is thought to be primarily demyelinating in nature, with rapid onset and often spontaneous complete resolution of signs and symptoms. In view of this, its rarity and relatively short disease process, clinical and pathological information are primarily lacking.

Transcranial magnetic stimulation (TMS) is a

Department of Neurology, Singapore General Hospital, Outram Road, Singapore 169608 Y L Lo

P Ratnagopal

Correspondence to: Dr Y L Lo

gnrlyl@sgh.gov.sg

Received 19 October 2000 and in revised form

9 February 2001

Accepted 30 March 2001
Case histories

PATIENT 1

A 39 year old women had no relevant medical history. She developed giddiness, diplopia, and limb numbness of acute onset, worsening over 3 days. A history of upper respiratory infection 1 week before admission was elicited. Physical examination on admission showed diminished eye movements in all directions, mild bilateral ptosis, areflexia, upper limb incoordination, and reduced limb sensation to touch and pain. No detectable motor weakness was present. Brain MRI and CSF examination were unremarkable. An antiGQ1b assay was positive. The patient's eye movements progressed to a near complete ophthalmoparesis over 2 days. Transcranial magnetic stimulation studies were performed on admission. She was discharged 1 week after admission with minimal improvement of eye movements. She continued to improve when reviewed fortnightly. At 1.5 months after admission, she recovered to having near complete eye movements and a repeat TMS study was done. At 7.5 months after admission, there was complete recovery of eye movements and ataxia but reflexes remained slightly diminished. A repeat TMS study was performed then.

\section{PATIENT 2}

A 55 year old women had no relevant medical history. She experienced double vision on waking as the only presenting complaint. Examination on admission disclosed abduction failure in the left eye with an otherwise unremarkable examination. On the second day of admission, abduction failure in the right eye and generalised hyporeflexia was elicited. This progressed to areflexia and total ophthalmoplegia by the 5th day of admission. In addition, there was mild upper limb incoordination and bilateral ptosis despite preserved motor power. Brain MRI was unremarkable. Examination of CSF was normal. She was tested positive for antiGQ1b antibody. Transcranial magnetic stimulation was performed on the 8th day of admission.

On review 3 weeks later, there was evidence of improved eye movements in all directions and normal coordination despite areflexia. The second outpatient review showed complete resolution of eye movements, ataxia, and a definite return of some tendon reflexes. A repeat TMS study was performed then. A third review, with a TMS study, was performed 6 months after admission. There was complete recovery of eye movements, ataxia, and normalisation of reflexes. 
PATIENT 3

A 70 year old man with no relevant medical history presented with a 4 day history of unsteady gait preceeded by an upper respiratory infection 1 week earlier. Examination showed reduced eye movements in all directions, limb incoordination, and generalised areflexia in the absence of detectable motor weakness. Brain MRI and CSF examination were unremarkable. He was tested positive for antiGQ1b antibody. Transcranial magnetic stimulation was performed on the 6th day of admission. On review I month later, clinical examination showed mildly improved eye movements and coordination despite generalised areflexia. A repeat TMS study was done. A second out patient review 8 weeks later showed near normalisation of eye movements, coordination, and return of tendon reflexes. A repeat TMS study was performed on this review date.

\section{Methods}

PATIENTS

All patients gave informed consent before TMS was performed. History of seizures, heart disease, and intracranial operations were excluded. Magnetic stimulation was performed with a Dantec Mag 2 Stimulator (Dantec Co, Denmark) with a Dantec $S 100$ circular $10 \mathrm{~cm}$ diameter coil generating up to 1.9 Tesla in output. Motor evoked potential (MEP) recordings were made with adhesive surface electrodes in the first dorsal interosseous and abductor hallucis muscles. The coil was positioned over the vertex to obtain consistent MEPs of maximum amplitude with the relevant muscle in slight contraction. Patients had multiple cortical stimulations, sometimes up to 20 for each muscle, to obtain distinctly recordable MEPs of consistent morphology. The average latency of the shortest of three most consistent responses was accepted. The minimum latency of $20 \mathrm{~F}$ responses was obtained; CMCT was calculated with the formula: MEP latency- $(\mathrm{F}$ latency $+M$ latency -1$) / 2$ in $m$ s. $^{6}$

Nerve conduction studies of the median, ulnar, sural, tibial, and peroneal nerves with relevant late responses were performed. Additional tests including evoked response studies were performed if clinically indicated.

\section{CONTROLS}

We performed CMCT measurements on 15 healthy volunteers with informed consent using the above technique. The ages ranged from 20 to 70 and included six men and nine women.

\section{Results}

NEUROPHYSIOLOGICAL STUDIES

Nerve conduction studies in patient 1 showed a mild sensory demyelinating polyneuropathy of the lower limbs and absent $\mathrm{H}$ reflexes. Auditory, median, and tibial somatosensory evoked responses were within normal limits. Nerve conduction studies in patient 2 were unremarkable. In patient 3, nerve conduction studies showed diminished lower limb sensory velocities, prolonged late responses, and absent $\mathrm{H}$ reflexes.
CENTRAL MOTOR CONDUCTION TIME

Patient 1 had severe prolongation of CMCTs to the upper limbs on presentation despite preservation of CMCTs to the lower limbs. The upper limb CMCTs showed marked reductions in the next two studies. Patient 2, similarly had prolonged CMCTs to the upper limbs in the initial study, although to a lesser extent. These two patients also showed a reducing trend towards the normal range by the second study. Again, CMCTs to the lower limbs were not prolonged and were relatively unchanged in subsequent studies. In patient 3 , CMCTs to the lower limbs were mildly prolonged in the initial study but showed gradual reductions in subsequent studies. Although upper limb CMCTs were not initially prolonged, there was evidence of gradual reduction in the later studies.

Mean (SD) for upper and lower limb CMCTs in control subjects were 5.85 (1.028) and 12.262 (1.875) ms respectively. The upper limit of normal at 2SDs were 7.745 and 16.011 ms.

The results are summarised in figures 1 and 2 for upper and lower limbs respectively; $M$ and $\mathrm{F}$ wave latencies are summarised in table 1.
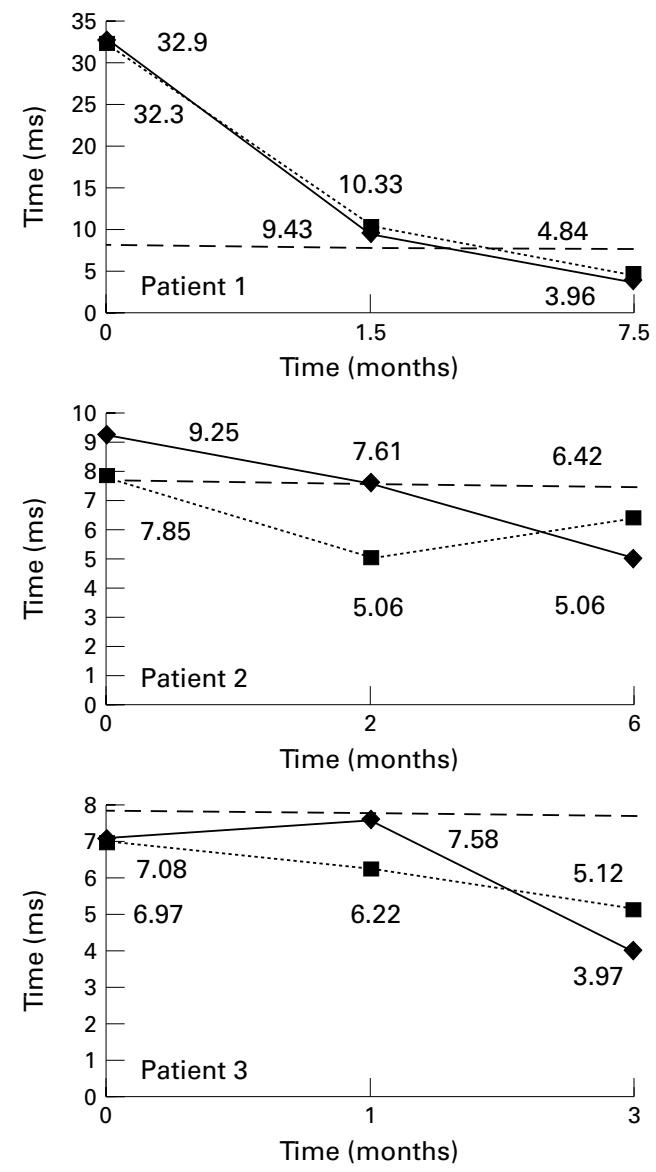

Figure 1 Dynamic CMCT changes in upper limb recordings. Dynamic CMCT reduction over time is evident in patients 1 and 2 . The dashed horizontal line represents the upper limit of CMCT at 2 SDs from controls. Continuous lines link left upper limb CMCTs. Dotted lines link right upper limb CMCTs. 

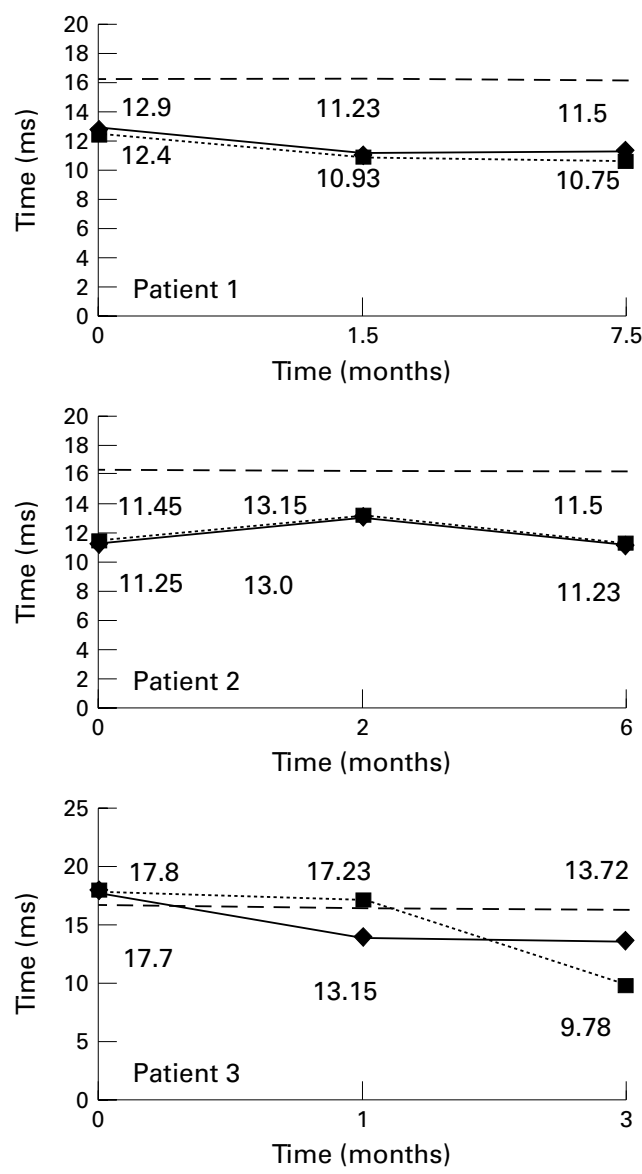

Figure 2 Dynamic CMCT changes in lower limb recordings. All symbols, axes, and lines as in figure 1. Dynamic reduction of CMCT over time is seen in patient

\section{Discussion}

The evidence for a CNS lesion in the Miller Fisher syndrome from clinical, immunological, and pathological aspects are few by contrast with the Guillain-Barré syndrome. Although a brain stem lesion has been suggested, direct pathological evidence of demyelination in the $\mathrm{CNS}^{78}$ is lacking. Serum antibody binding to human and mouse cerebellum has been implicated as a cause for ataxia, ${ }^{9}$ but current immunological evidence has mainly shown binding to cranial nerves involved in eye movements. ${ }^{10}$ Magnetic resonance imaging studies have shown enhancement in the lumbosacral root, cauda equina, facial nerve, and trigeminal nerves. ${ }^{11-13}$ Isolated cases of medullary, ${ }^{14}$ midbrain, ${ }^{15}$ and pontine abnormalities ${ }^{16}{ }^{17}$ have also been reported, but the nature of these lesions remains obscure.

Neurophysiological variables have not been used extensively in patients with this condition. Separate studies gave conflicting results on brain stem auditory evoked potentials, whereas blink reflex studies have not been useful for electrophysiological localisation. ${ }^{18-20}$ Peripheral nerve conduction studies in general do not differ significantly from those in patients with Guillain-Barré syndrome. $^{21} 22$

Central motor conduction time is an established variable in assessing the speed of corticospinal tract conduction from the motor cortex to the spinal motor neurones. It is used in the diagnosis and follow up of central demyelinating disorders, most notably multiple sclerosis. ${ }^{23}$ Although it can be suggested that prolonged MEP latencies are the result of abnormal $\mathrm{F}$ wave latencies, our study showed that they are relatively stable and not significantly prolonged (fig 3), with the exception of patient 3 in the later studies (table 1). Also, the mathematical method of CMCT calculation has taken into account prolonged $\mathrm{F}$ and $\mathrm{M}$ latencies.

Our findings of dynamic reduction of CMCTs in tandem with clinical improvement support the hypothesis of a transient neuronal insult. The lesion or lesions in question are also likely to be central and variable in localisation, as evidenced by separate involvement of upper and lower limb central connections in different patients. This finding is also in keeping with radiological reports of MRI abnormalities in varied locations in separate patients with this disease. AntiGQ1b positivity in all three patients lends supportive evidence for a diffuse immunological disease process. It is possible that the ongoing immunological attack is patchy, multifocal, or variable in location.

Although isolated studies have reported MRI abnormalities, this has been the exception rather than the rule, as seen in this study. The relevance of the absence of radiologically detectable lesions in the presence of electrophysiological abnormalities is uncertain, but could imply the presence of a transient non-structural immunological attack causing blockage of impulse transmission with no permanent axonal damage. All three patients hence showed complete recovery on follow up. The concept of reversible conduction disturbances has been studied for peripheral nerves in the Guillain-Barré syndrome, in terms of

Table 1 Summary table of $M$ wave latency, $F$ wave latency, and CMCT of all patients in each follow up study. All values are in ms. The first column shows right sided values of each measurement

\begin{tabular}{|c|c|c|c|c|c|c|c|c|c|c|c|c|c|c|c|c|c|c|}
\hline & \multicolumn{18}{|c|}{ Study } \\
\hline & \multicolumn{6}{|l|}{1} & \multicolumn{6}{|l|}{2} & \multicolumn{6}{|l|}{3} \\
\hline & \multicolumn{2}{|c|}{$M$ Wave } & \multicolumn{2}{|c|}{ F Wave } & \multicolumn{2}{|c|}{$C M C T$} & \multicolumn{2}{|c|}{$M$ Wave } & \multicolumn{2}{|c|}{$F$ Wave } & \multicolumn{2}{|l|}{$C M C T$} & \multicolumn{2}{|c|}{$M$ Wave } & \multicolumn{2}{|c|}{ F Wave } & \multicolumn{2}{|l|}{$C M C T$} \\
\hline \multicolumn{19}{|l|}{ Upper limb: } \\
\hline Patient 1 & 3.9 & 3.4 & 27.8 & 27.1 & 32.3 & 32.9 & 2.9 & 3.9 & 25.1 & 25.9 & 10.33 & 9.43 & 4.1 & 3.7 & 25.5 & 26.1 & 4.84 & 3.96 \\
\hline Patient 2 & 2.5 & 2.5 & 25.4 & 24.6 & 9.25 & 7.45 & 3.9 & 3.5 & 27.6 & 27.6 & 5.06 & 7.61 & 3.7 & 3.9 & 24.3 & 27.6 & 6.42 & 5.06 \\
\hline Patient 3 & 4.1 & 3.5 & 31.4 & 31.0 & 6.97 & 7.08 & 4.5 & 4.1 & 32.5 & 29.4 & 6.22 & 7.58 & 4.5 & 4.3 & 34.7 & 33.3 & 5.12 & 3.97 \\
\hline \multicolumn{19}{|l|}{ Lower limb: } \\
\hline Patient 1 & 7.8 & 6.0 & 49.9 & 50.2 & 12.9 & 12.4 & 5.5 & 5.6 & 47.4 & 46.7 & 10.93 & 11.23 & 5.2 & 5.6 & 49.3 & 47.4 & 10.75 & 11.5 \\
\hline Patient 2 & 3.2 & 3.2 & 46.7 & 47.9 & 11.45 & 11.25 & 4.5 & 4.5 & 50.2 & 48.0 & 13.15 & 13.0 & 4.1 & 3.7 & 46.4 & 46.1 & 11.5 & 11.23 \\
\hline Patient 3 & 7.8 & 5.9 & 57.6 & 55.7 & 17.8 & 17.7 & 7.3 & 7.8 & 58.0 & 61.5 & 17.23 & 13.98 & 7.5 & 6.5 & 62.7 & 65.5 & 9.78 & 13.72 \\
\hline
\end{tabular}



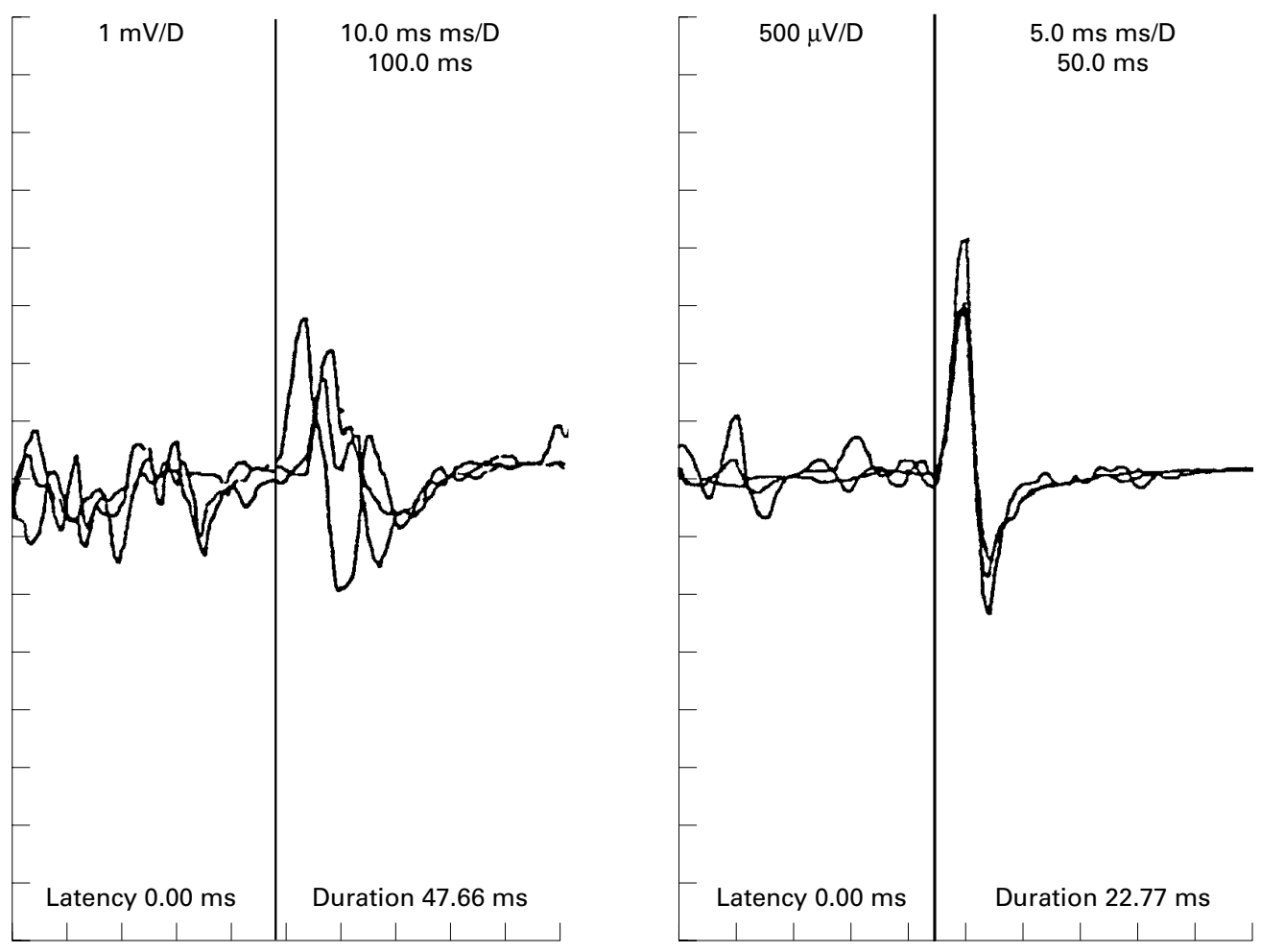

Figure 3 MEP recordings of patient 1 (left) and normal control (right) from the right first dorsal interosseous comprising three superimposed reproducible MEPs. Patient MEP is delayed and temporally dispersed in morphology. Gain and sweeps as indicated in tracings. Absolute MEP latencies are given at the bottom of each tracing.

antibody facilitated immune attack ${ }^{24}$ and serial electrophysiological studies. ${ }^{25-27}$ A recent study suggested antiGQ1b-mediated blockage of acetylcholine release from motor nerve terminals in a patient with Miller Fisher syndrome. ${ }^{28}$ It is hypothesised that similar mechanisms could be responsible for the electrophysiological findings in the central corticospinal pathways in our cases.

Although all three patients had electrophysiological evidence of corticospinal tract conduction abnormalities, none had demonstrable upper motor neuron-type deficits clinically. It is argued that central motor conduction time prolongation probably represents desynchronisation of descending volleys during transcranial magnetic stimulation caused by a transient immunologically mediated "functional demyelinating process", which is not sufficiently translated into obvious clinical weakness or upper motor release signs.

In conclusion, we have provided supportive evidence of central, transient, and reversible corticospinal tract dysfunction in the Miller Fisher syndrome. The tendency to clinical recovery, absence of radiological abnormalities, and rapid reduction of central motor conduction times favour a non-axonal disease process.

1 Sauron B, Bouche P, Canthala HP, et al. Miller Fisher syndrome: clinical and electrophysiologic evidence of peripheral origin in 10 cases. Neurology 1984;43:953-6.

2 Hughes RA, Rees JH. Guillain-Barré syndrome. Curr Opin Neurol 1994;7:386-92.

3 Mizoguchi K. Anti-GQ1b antibody activities related to the Mizoguchi K. Anti-GQ1b antibody activities related to the
severity of Miller Fisher syndrome. Neurol Res 1998;20: severity
4 Van der Meche FG, Visser LH, Jacobs BC, et al. Guillain-Barré syndrome: multifactorial mechanisms versus defined subgroups. F Infect Dis 1997;176(suppl 2):99-102.

5 Barontini F, Di Lollo S, Maurri S, et al. Localisation of the pathological process in Miller Fisher syndrome. Ital f Neurol Sci 1992;13:221-5.

6 PM Rossini, F Pauri. Central motor conduction time studies. In: Paulus W, et al, ed. Transcranial magnetic stimulation. Amsterdam: Elsevier, 1999:199-211.

7 Al-Din AN, Anderson M, Bickerstaff ER, et al. Brainstem encephalitis and the syndrome of Miller Fisher. Brain 1982;105:481-95.

8 Maier H, Schmidbauer M, Pfausler B, et al. Central nervous pathology in patients with the Guillain-Barré syndrome. Brain 1997;120:451-64.

9 Inoue A, Koh C, Iwahashi T. Detection of serum anticerebellar antibodies in patients with Miller Fisher syndrome. Eur Neurol 1999;42:230-4.

10 Kusunoki S. Antiganglioside antibodies in the pathogenesis of autoimmune neuropathies. Rinsho Shinkeigaku 1999;39: of autoin.

11 Pedotti R, Carpo M, Lucchi S, et al. Lumbosacral root and facial nerve enhancement in Miller Fisher syndrome. $\mathcal{F}$ Neurol 1998;245:753-4.

12 Nagaoka U, Kato T, Kurita K, et al. Cranial nerve enhancement on three-dimensional MRI in Miller Fisher syndrome. Neurology 1996;47:1601-2.

13 Farah S, Khuraibet AJ, Montaser MA, et al. Miller Fisher syndrome: unique involvement of ophthalmic division of syndrome: unique involvement of ophthalmic division of
trigeminal nerve. Clin Neurol Neurosurg 1995;97:328-31.

14 Urushitani M, Udaka F, Kameyama M. Miller FisherGuillain-Barré overlap syndrome with enhancing lesions in the spinocerebellar tracts. $\mathcal{F}$ Neurol Neurosurg Psychiatry the spinocerebel $1995 ; 58: 241-3$.

15 Hatanaka T, Higashino H, Yasuhara A, et al. Miller Fisher syndrome: etiological significance of serial blink reflexes and MRI study. Electromyogr Clin Neurophysiol 1992;32: $317-9$

16 Giroud M, Mousson C, Chalopin JM, et al. Miller Fisher syndrome and pontine abnormalities on MRI: a case report. F Neurol 1990;237:489-90.

17 Taphoorn MJ, Uitehaag BM, Lanting P. A brainstem lesion in the Miller Fisher syndrome demonstrated by CT and MRI. $\mathcal{F}$ Neurol 1991;238:243.

18 Fantin A, Valenzuela R, Luco C, et al. Auditory evoked potentials in Fisher's syndrome. A clinical case. Rev Med Chil 1991;119:798-802.

19 Jamal GA, Ballantyne JP. The localisation of the lesion in patients with acute ophthalmoplegia, ataxia and areflexia
(Miller Fisher syndrome), A serial multimodal neurophysiological study. Brain 1988;111:95-114. 
20 Goldberg-Stern H, Melamed E, Gadoth N. Abnormal evoked potentials in Miller Fisher syndrome: further evidence of combined peripheral and central demyelinaPsychiatry 1994;57:506.

21 Jamal GA, MacLeod WN. Electrophysiologic studies in Miller Fisher syndrome. Neurology 1984;34:685-8.

22 Guiloff RJ. Peripheral nerve conductions in Miller Fisher syndrome. F Neurol Neurosurg Psychiatry 1977;40:801-9.

23 Cowan JMA, Rothwell JC, Dyck JPR, et al. Abnormalities in central motor conduction in multiple sclerosis. Lancet 1984;ii:304-7.

24 Kuwabara S, Yuki N, Koga M, et al. IgG anti-GM1 antibody is associated with reversible conduction failure and axona degeneration in Guillain-Barré syndrome. Ann Neurol 1998;44:202-8

25 Berger AR, Logigian EL, Shahani BT. Reversible proximal conduction block underlies rapid recovery in GuillainBarré syndrome. Muscle Nerve 1988;11:1039-42

26 Sumner A, Said G, Idy I, et al. Electrophysiological effects of the injection of Guillain-Barré sera in the sciatic nerve of the rat. Rev Neurol (Paris) 1982;138:17-24.

27 Sumner AJ. The physiological basis for symptoms in Guillain-Barré syndrome. Ann Neurol 1981;9:28-30.

28 Uncini A, Lugaresi A. Fisher syndrome with tetraparesis and antibody to GQ1b: evidence for motor nerve terminal block. Muscle Nerve 1999;22:640-4.

\section{HISTORICAL NOTE}

\section{The Lewy body}

James Parkinson noted:

"A diseased state of the medulla spinalis, in that part which is contained in the canal, formed by the superior cervical vertebrae, and extending, as the disease proceeds, to the medulla oblongata . . . is the proximate cause."

In the late 19 th century, because knowledge of the pathophysiology of the basal ganglia was imprecise, Gowers and others implicated the motor cortex as the source of Parkinson's disease. Edouard Brissaud (a neurologist's neurologist) in 1894 thought that the site of Parkinson's disease must be peduncular or subthalamic, rejecting prevalent theories that it was muscular or a neurosis. He reported a parkinsonian syndrome caused by a tuberculoma of the substantia nigra and concluded.

"The locus niger might well be its anatomical substratum."

In a vital paper, Tretiakoff in 1919 examined the brains of nine parkinsonian patients. $\mathrm{He}$ was the first to state that substantia nigra esions were important in both Parkinson's disease and in postencephalitic patients, a view supported by Greenfield. Tretiakoff ${ }^{3}$ noted in particular, reduced numbers of pigmented cells in the locus niger, which he related to a disorder of muscular tone in Parkinson s disease. He also found peculiar concentric inclusions in the cytoplasm of these nigral cells. A more detailed study of mid-brain neuropathology by Foix and Nicolesco ${ }^{4}$ in 1925 completed the anatomical picture, which so frustratingly had eluded James Parkinson.

However, these inclusion bodies had already been described by Friederich Lewy (1885$1950)^{5}$ who discovered them in $1912^{6}$ while working in Alzheimers laboratory. ${ }^{7}$ They proved to be the hallmark of Parkinson's disease. $^{8}$
Lewy described chronic cell atrophy and glial overgrowth in the putamen and globus pallidus, with reduction of fibres in the ansa lenticularis. These he likened to the lesions of senility. Later, in 1923, he observed ${ }^{9}$ that most of the atrophic cells showed a senile fibrillar change, and emphasised the great cellular loss in the pallidum, the dorsal nucleus of vagus (vegetative oblongata nucleus) when there was tremor of the larynx, and also stated that cells of the substantia nigra are regularly involved. The characteristic, but non-diagnostic Lewy bodies remain the major features ${ }^{10}$ on microscopic examination. ${ }^{11}$ Lewy found them most strikingly in the nucleus basalis, substantia innominata, and in the dorsal motor nucleus of the vagus, but less often in the substantia nigra. Subsequent studies showed them in neurons carrying neuromelanin pigment: the locus coeruleus, autonomic ganglia, amygdala, and hypothalamus. They can be seen throughout the cortex in smaller numbers.

A cautious observer, Lewy wrote:

"They (intracellular inclusions) are simply findings which I up till now have found in all cases of paralysis agitans that

I have examined, but which were absent

in the other (control) cases."

Lewy knew little of the biology of neuromelanin. We now know it may be the result of breakdown of intracellular catecholeamines, including dopamine, but whether it protects the cell or is itself toxic when it accumulates is uncertain. It is of interest that neuromelanin is preserved in albinos. Lewy bodies are circular eosinophilic structures with a dense protein core surrounded by a peripheral halo located within the cytoplasm of neurons The ultrastructural appearance is like a sunflower with a dense central core of circular shaped structures and a rim of radiating filaments (7 to $20 \mathrm{~nm}$ in diameter), the largest filaments at the periphery, which corresponds to the halo. Their size and number vary. Lewy bodies result from neuronal degeneration, with accumulated altered cytoskeletal elements that stain with antiubiquitin antibodies and antibodies to neurofilaments.
Lewy stressed that they were not confined to Parkinson's disease. About 5\% to $10 \%$ ofasymptomatic people have Lewy bodies, usually in the substantia nigra. ${ }^{12}$ Later work showed that they occur in other neurodegenerative disorders including the nosologically ambiguous dementia with Lewy bodies. ${ }^{13}$

J M S PEARCE

304 Beverley Road, Anlaby, Hull HU10 7BG, UK jmspearce@freenet.co.uk

1 Parkinson J. An essay on the shaking palsy. Chapter 4. London: Sherwood, Neely, and Jones, 1817.

2 Brissaud E. Leçons sur les maladies nerveuses. Paris: Masson, 1895:469-501.

3 Tretiakoff C. Contributions a l'etude de l'anatomie pathologique du locus niger de soemmering avec quelques deductions relatives a la pathogenie des troubles de tomus musculaire et de la maladie de Parkinson Paris: Thesis, 1919.

4 Foix C, Nicolesco J. Anatomie cerebrale: les noyaux gris Centraux et la region noyaux gris Centraux et la region
Mesencephalo-soux-optique. Paris: Masson, 1925.

5 Anon. Biography of Lewey. Archives of Neurology and Psychiatry, Chicago 1951;66:114-5.

6 Lewy FH. Paralysis agitans. In: Lewandowsky $\mathrm{M}$, ed. Pathologische Anatomie. Handbuch der Neurologie. Berlin: Springer-Verlag, 1912: 920-33

7 Lewy FH Zur pathologischen Anatomie der Paralysis Agitans. Deutsche Zeitschrift fur Nervenheilkunde 1913;50:50-5.

8 Forno LS. Pathology of Parkinson's disease: the importance of the substantia nigra and Lewy bodies. In: Stern G. Parkinson s disease. London: Chapman Hall, 1990:185-238.

9 Lewy FH. Die Lehre vom tonus und der Bewegung zugleich systematische Untersuchungen zur Klijik, Physiologie, Pathologie und Pathogenese der Paralysis agitans. Berlin: Springer Verlag, 1923.

10 Ferman TJ. Dementia with Lewy bodies: a Ferman TJ. Dementia with Lewy bodies: a
review of clinical diagnosis, neuropathology and management options. http:// and management options. dcmsonline.org/_vti_bin/shtml.dll/jaxmedicine/February2000/lewybodies.htm/map

11 Forno LS. Pathology of Parkinson's disease: the importance of the substantia nigra and Lewy bodies. In: Stern GM, ed. Parkinson's disease. Baltimore, MD: Johns Hopkins University Press, 1990:185-238.

12 Hansen LA, Galasko D. Lewy body disease. Curr Opin Neurol Neurosurg 1992;5:889-894.

13 Litvan I, MacIntyre A, Goetz CG, et al. Accuracy of the clinical diagnoses of Lewy body disease, Parkinson disease, and dementia. Arch Neurol 1998;55:969-78. 